\title{
MODEL CALIBRATION OF BETATRON FUNCTIONS AND PHASE IN THE SPring-8 STORAGE RING
}

\author{
G. Liu \\ Shanghai Institute of Nuclear Research, Chinese Academy of Sciences \\ P.O.Box 800-204, Shanghai 201800, China \\ K. Kumagai, N. Kumagai, H. Ohkuma, K. Soutome* , M. Takao, H. Tanaka \\ SPring-8, Mikazuki, Sayo, Hyogo 679-5198, Japan \\ defined by

$$
\begin{gathered}
g_{i}^{\mathrm{BPM}}=\frac{\text { actual shift of beam position }}{\text { shift of BPM readout }} \\
g_{j}^{\mathrm{ST}}=\frac{\text { actual kick angle }}{\text { required kick angle }}
\end{gathered}
$$

\section{Abstract}

We developed a model calibration method to measure the betatron function and phase of a large storage ring. The results of applying this method to the SPring- 8 storage ring will be presented.

\section{MODEL CALIBRATION METHOD}

In third generation light sources, such as the SPring-8 storage ring, it is important to measure various errors which deteriorate the quality of a stored beam. For example, focusing errors coming from quadrupole field errors or from sextupole misalignments generate distortions of linear optics, coupling of horizontal and vertical betatron oscillations and spurious dispersion in the vertical direction.

A most conventional method of measuring the betatron function of a storage ring will be to vary the strength of a quadrupole magnet and detect the betatron tune shift. This method however requires a magnet power supply system that allows us to control the strength of each quadrupole magnet independently. Furthermore, the betatron function is obtained only at the position of quadrupole magnets.

Another method also used commonly is to measure the response matrix of a ring and use this data to fit the betatron function and phase. Here, the response matrix $R_{i j} \equiv$ $R\left(s_{i}, s_{j}\right)$ is defined as the orbit change at the BPM position $s_{i}$ for a unit kick given at the steering magnet position $s_{j}$. This method has a merit that BPM offsets are automatically canceled and the betatron function and phase are obtained consistently at the positions of BPMs and steering magnets. However, the accuracy of measurements could be dominated by calibration errors of BPMs and steering magnets.

We then developed a method by combining the response matrix and a model of the ring simplified suitably for the SPring-8 storage ring. The point of our approach (Model Calibration Method: MCM[1],[2],[3]) is that as fitting parameters of the ring we do not use the betatron function itself nor individual focusing errors generated by each qaudrupole or sextupole magnet. Instead, we use effective focusing errors given by integrating individual errors over some magnets. In addition to such focusing errors, we introduce calibration factors of BPMs and steering magnets

\footnotetext{
*Corresponding author; Email: soutome@ @pring8.or.jp
}

and the energy shift $\delta_{j}$ generated by the horizontal unit kick by the $j$-th steering magnet located in a dispersive section. Note that this energy shift causes orbit shifts at BPM positions and the term $\eta_{i} \delta_{j}$ must be included in the model response matrix $R_{i j}^{\text {(model) }}$, where $\eta_{i}$ is the dispersion function measured at the $i$-th BPM. The best values for these fitting parameters are obtained by minimizing

$$
\chi^{2} \equiv \sum_{i, j} \frac{\left(R_{i j}^{(\text {model })}-R_{i j}^{(\text {measured })}\right)^{2}}{\sigma_{i}^{2}}
$$

where $\sigma_{i}$ is the rms noise of the $i$-th BPM. For calculating $R_{i j}^{\text {(model) }}$ as a function of the fitting parameters, we developed a simulation code based on the RACETRACK[4].

As seen from Eq. 3 the accuracy of fitting largely depends on the accuracy of BPMs. In the SPring-8 storage ring the value of $\sigma_{i}$ has been measured at each BPM by using a stored beam and the results show that $\sigma_{i}$ takes a value between $2 \mu \mathrm{m}$ and $5 \mu \mathrm{m}$, depending on the BPM position, in both horizontal and vertical directions.

\section{MODEL}

In the SPring- 8 storage ring there are 288 BPMs, 285 horizontal and 283 vertical steering magnets, 480 quadrupole magnets and 336 sextupole magnets. Then, a direct application of MCM requires a huge memory size and a very fast $\mathrm{CPU}$ to computers. Furthermore, as will be shown later, an individual focusing error that each quadrupole or sextupole magnet generates can not be distinguished by the present resolution of BPMs.

To overcome these difficulties, we introduced a model of the ring in which a suitable number of integrated focusing errors are distributed. The total number of errors was chosen to be 240, which should be compared with the total number of quadrupole and sextupole magnets (816). This number of 240 was fixed with the help of computer simulations in the following way: First, we checked how fine we can distinguish the focusing errors by using the present 
BPM system. The results show that the expected resolution is $1.3 \times 10^{-3} \mathrm{~m}^{-1}$ when the response matrix data is taken by using a single kick of $0.05 \mathrm{mrad}$. This resolution is too coarse to distinguish an expected individual error of about $2 \times 10^{-4} \mathrm{~m}^{-1}$ that each quadrupole or sextupole magnet will generate. This result indicates the necessity of introducing integrated focusing errors into our model.

We then searched an optimum number and distribution of integrated focusing errors, taking account of the betatron phase advance in both horizontal and vertical directions. Figure 1 shows how we can improve the accuracy of fitting of the betatron function and phase by increasing the number of distributed focusing errors in a unit cell. Note that even if we increase the number of errors per unit cell beyond 5 , the accuracy of fitting is not improved any more. This level of accuracy is determined by resolutions of the present BPM system. We then fixed the number of errors per unit cell to be 5 . We remark that the lattice structure of the SPring- 8 storage ring is of the double-bend achromat type, and within a unit cell there are three girders separated by two bending magnets. The betatron phase advance within the first and third girder is small and a single error source was assigned to these. On the other hand, the betatron phase advance within the second girder is large and we assigned three error sources to this. In such a model, the accuracy of determining the betatron functions is expected to be better than $1 \%$ as seen from Figure 1 .

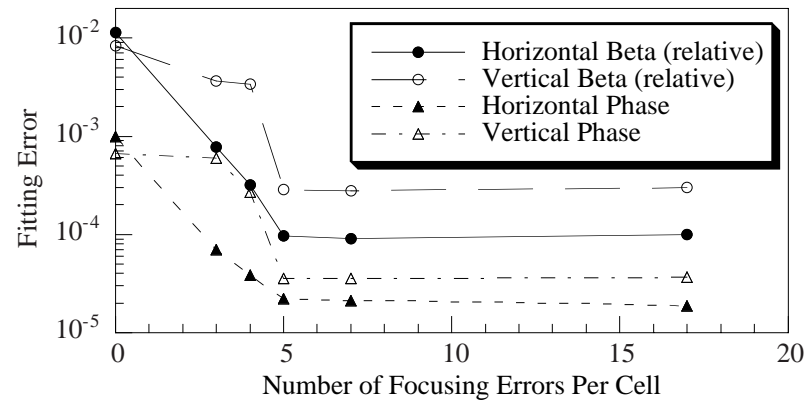

Figure 1: The accuracy of fitting as a function of the number of integrated focusing errors per unit cell.

We note that, in addition to the errors randomly distributed along the ring, there can be systematic errors arising from, e.g., incompleteness of the model. An estimation of such errors is generally difficult and the model finally obtained by MCM should be tested by some means.

We also remark that the coupling ratio of horizontal and vertical betatron oscillations has been deduced experimentally [5] and the results show that the ratio is much smaller than $1 \%$. We then neglected the coupling.

\section{RESULTS AND DISCUSSION}

All elements of the response matrix were measured in 11 hours by giving a single kick of $0.05 \mathrm{mrad}$ to the stored beam. This kick angle was determined by requiring that a nonlinear behavior of the ring is sufficiently suppressed and the difference of two orbits is measured with enough resolutions. Since the total amount of the data was huge, we divided it into four parts according to the position of excited steering magnets. A quarter of the data was then used for applying MCM and this procedure was repeated four times to cover the whole ring.

In Fig. 2 we show correlation between measured and model response matrix elements. The model response matrix was calculated in two ways - with (right) and without (left) application of MCM. We see that the model response matrix has been improved by applying MCM: the rms value of the difference between measured and model response matrix elements is reduced from $81 \mu \mathrm{m}$ to $3.3 \mu \mathrm{m}$ in the horizontal direction and from $85 \mu \mathrm{m}$ to $3.8 \mu \mathrm{m}$ in the vertical direction. This means that MCM almost converged to the level of the present resolution of BPMs.
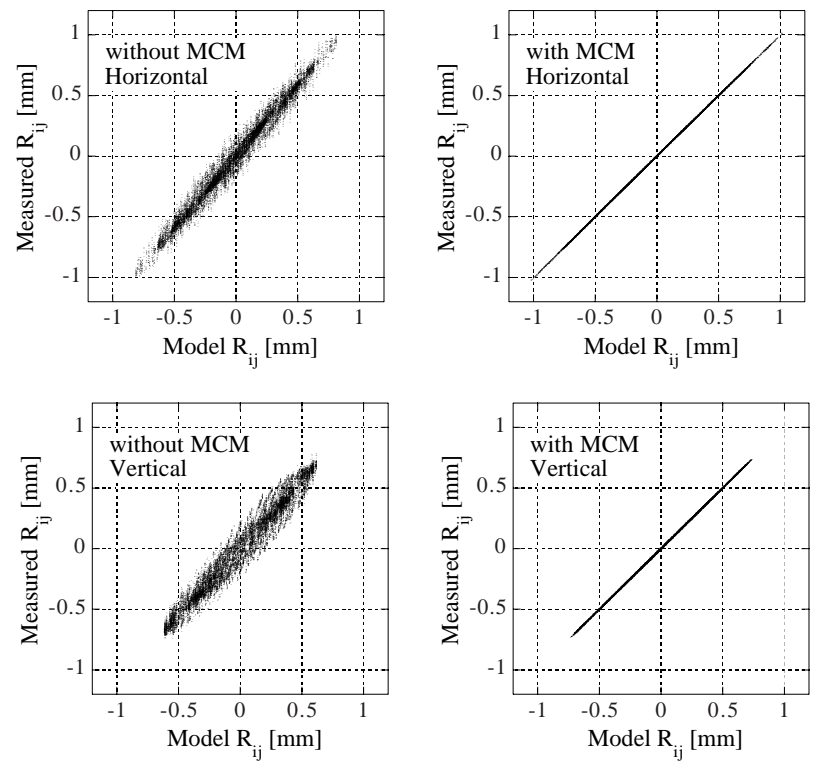

Figure 2: Correlation between measured and model response matrix elements.

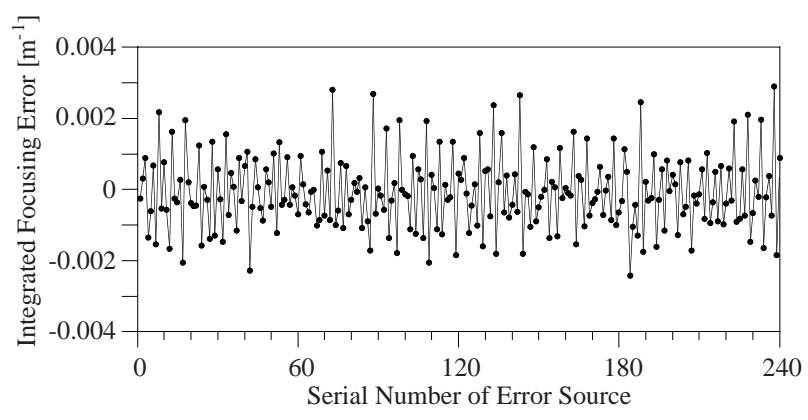

Figure 3: Focusing error distribution of the ring.

A distribution of effective focusing errors in the ring and calibration factors of BPMs and steering magnets were estimated, and the results are shown in Fig. 3, Fig. 4 and Fig. 5, respectively.

In Fig. 5, we see that some vertical steering magnets have a large deviation of the gain factor from unity. The reason 


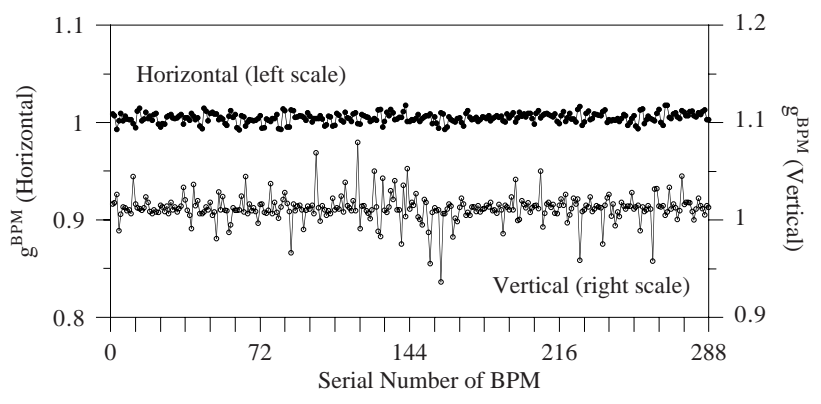

Figure 4: Gain factors of BPMs.

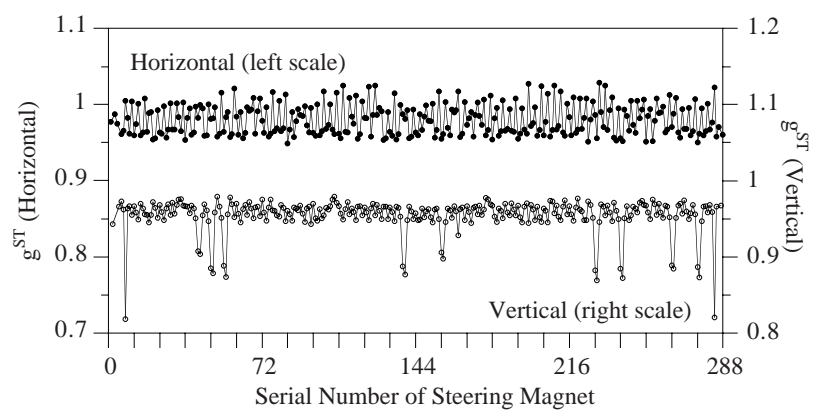

Figure 5: Gain factors of steering magnets.

for this was found to be the effect of a neighboring magnet settled close to these steering magnets: a fast kicker magnet or an auxiliary corrector magnet for an insertion device. This was checked experimentally by making a local bump orbit and observing its leakage with and without taking account of the gain factors. When we took account of the gain factors of the steering magnets, the leakage of a bump orbit was reduced as expected.

A part of the betatron function is shown in Fig. 6 by solid curves. For comparison, we also show model calculations without applying MCM by dashed curves. We see that distortions of the solid curves are very small. The horizontal and vertical betatron tunes of the ring corresponding to the solid curves are 51.235 and 16.308 , respectively, which agree well with experimental values 51.234 and 16.307.

To check the betatron functions thus obtained, we measured their values at some quadrupole magnets by changing their strength independently and detecting the betatron tune shifts. In Fig. 7 measured values at 10 quadrupole magnets in one unit cell are plotted and compared with the MCM results. In addition to these local measurements, we also measured average values by changing the strength of 48 quadrupole magnets at the same rate, family by family. The results are shown in Fig. 8. The agreement between measured and MCM values is quite satisfactory.

Acknowledgment: The authors would like to thank Drs. S.Daté, K.Fukami, M.Masaki, T.Ohshima, S.Sasaki, M.Shoji, S.Takano and K.Tamura for useful discussions and cooperation in machine operations.
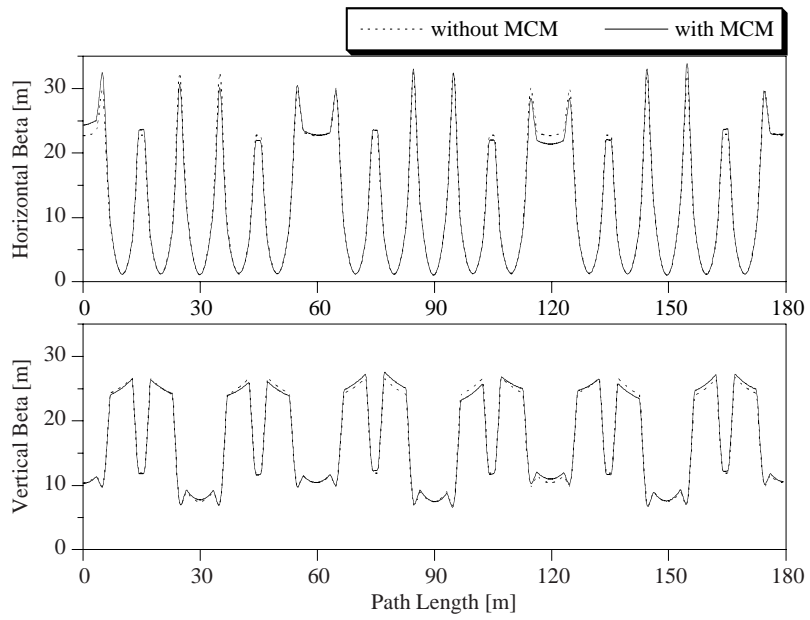

Figure 6: Betatron functions over 6 cells.

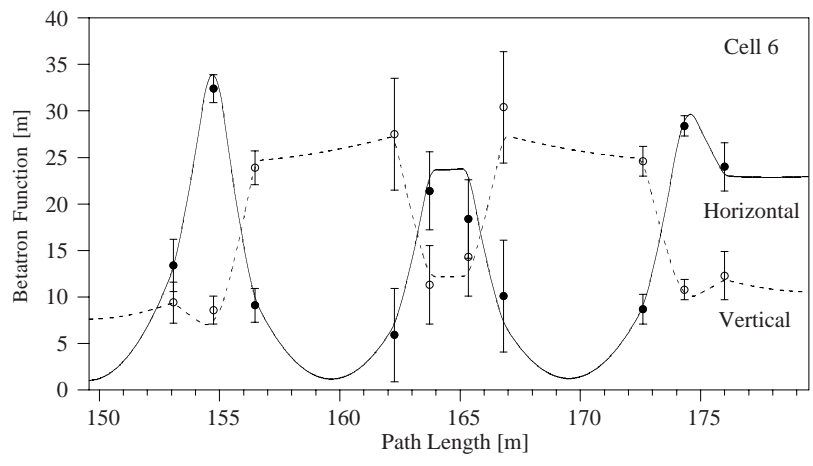

Figure 7: Betatron functions in one unit cell.

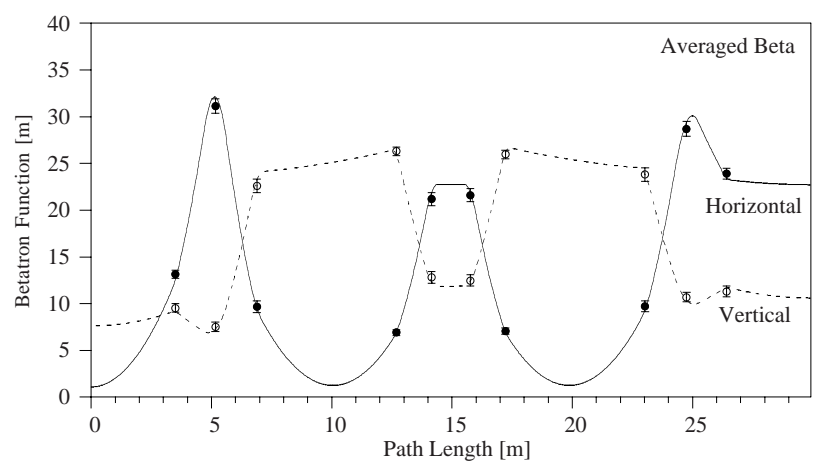

Figure 8: Averaged betatron functions.

\section{REFERENCES}

[1] G. Liu, et.al., SPring-8 Ann. Report 1997, p.174.

[2] W.J. Corbett, M.J. Lee and V. Ziemann, SLAC-PUB-6111 (1993).

[3] J. Safranek, Nucl.Instr.Meth. A388(1993)27.

[4] A. Wrulich, DESY 84-026 (1984).

[5] M. Takao, et.al., "Estimation of Betatron Coupling and Vertical Dispersion for SPring-8 Storage Ring", presented at this conference. 